\title{
Toxin-induced pore formation is hindered by intermolecular hydrogen bonding in sphingomyelin bilayers
}

Sara García-Linares, ${ }^{1,2}$ Juan Palacios-Ortega, ${ }^{1,2}$ Tomokazu Yasuda, ,,3 Mia Åstrand, ${ }^{2}$ José G. Gavilanes, ${ }^{1}$ Álvaro Martínez-del-Pozo, ${ }^{1}$ and J.Peter Slotte ${ }^{2 *}$

${ }^{1}$ Departamento de Bioquímica y Biología Molecular I, Universidad Complutense, Madrid, Spain ${ }^{2}$ Biochemistry, Faculty of Science and Engineering, Åbo Akademi University, Turku, Finland ${ }^{3}$ Department of Chemistry, Graduate School of Science, Osaka University, Osaka, Japan

*Corresponding author (email:jpslotte@abo.fi)

Short title: Intermolecular hydrogen bonding

Key words: Permeabilization, surface plasmon resonance, sphingomyelinase, sticholysin

Abbreviations: BA - benzyl alcohol; DPH - diphenyl hexatriene; EqtII - equinatoxin II; LUV large unilamellar vesicle; FraC - fragaceatoxin; OSM - $N$-oleoyl-D-erythro-sphingomyelin; POPC - 1-palmitoyl-2-oleoyl-sn-glycero-3-phosphocholine; PSM - N-palmitoyl-D-erythrosphingomyelin; SM - sphingomyelin; StnI - sticholysin I; StnII - sticholysin II 
Highlights

- Sphingomyelin and dihydro sphingomyelin hydrogen bond differently

- Sticholysin binds to sphingomyelin and forms pores in fluid bilayers

- Sticholysin failed to form pores in fluid dihydro sphingomyelin bilayers

- Strong intermolecular hydrogen bonding appeared to hinder pore formation

Graphical abstract:

Inhibitory effects of sphingomyelin hydrogen bonding on sticholysin-induced pore formation
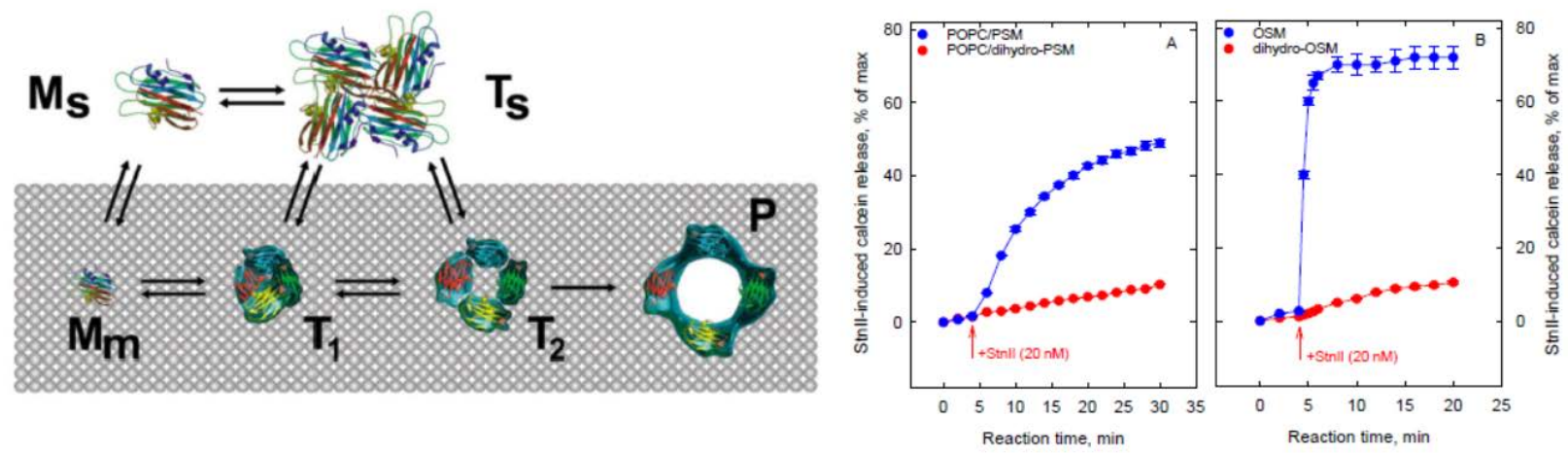
ABSTRACT Sticholysin I and II (StnI and StnII) are pore-forming toxins that use sphingomyelin (SM) for membrane binding. We examined how hydrogen bonding among membrane SMs affected the StnI- and StnII-induced pore formation process, resulting in bilayer permeabilization. We compared toxin-induced permeabilization in bilayers containing either SM or dihydro-SM (lacking the trans $\Delta^{4}$ double bond of the long-chain base), since their hydrogen-bonding properties are known to differ greatly. We observed that whereas both StnI and StnII formed pores in unilamellar vesicles containing palmitoyl-SM or oleoyl-SM, the toxins failed to similarly form pores in vesicles prepared from dihydro-PSM or dihydro-OSM. In supported bilayers containing OSM, StnII bound efficiently, as determined by surface plasmon resonance. However, StnII binding to supported bilayers prepared from dihydroOSM was very low under similar experimental conditions. The association of the positively charged StnII (at pH 7.0) with unilamellar vesicles prepared from OSM led to a concentrationdependent increase in vesicle charge, as determined from zeta-potential measurements. With dihydro-OSM vesicles, a similar response was not observed. Benzyl alcohol, which is a small hydrogen-bonding compound with affinity to lipid bilayer interfaces, strongly facilitated StnIIinduced pore formation in dihydro-OSM bilayers, suggesting that hydrogen bonding in the interfacial region originally prevented StnII from membrane binding and pore formation. We conclude that interfacial hydrogen bonding was able to affect the membrane association of StnI- and StnII, and hence their pore forming capacity. Our results suggest that other types of protein interactions in bilayers may also be affected by hydrogen-bonding origination from SMs. 


\section{Introduction}

Actinoporins are a group of soluble monomeric peptide toxins that have evolved to use sphingomyelin (SM) as their membrane recognition target [1-7]. These actinoporins include toxins such as sticholysin I and II (StnI and StnII, from Stichodactyla helianthus [8-10]), equinatoxin II (EqtII, from Actina equina [11]), and fragaceatoxin C (FraC, from Actina fragacea [12]). The water-soluble structure of the toxins is known in detail [13-16]. They have a $\beta$-sandwich motif consisting of $10 \beta$-strands flanked by two $\alpha$-helices, which interact with both sides of the $\beta$-sandwich. For the actinoporins, it is generally believed that monomeric peptides bind to the membrane interface (aided by SM) before oligomerization takes place, and a functional pore is finally formed $[6,7]$.

The effects of membrane properties on toxin binding and pore formation by actinoporins have received a lot of attention over the years (for recent reviews, see $[6,7,17]$ ). Quite a lot is known about how membrane and SM properties affect pore formation by different actinoporins. In the absence of cholesterol, pore formation by StnII requires the hydrogenbonding competent SM [18]. Indeed, all examined actinoporins appear to require the presence of SM in their target membranes [8,18-20]. However, under some conditions, both EqtII and StnII have been shown to increase bilayer permeability in the absence of SM $[8,20]$. Cholesterol has been shown to affect the pore-formation kinetics and efficiency of actinoporins $[21,22]$. The actual mechanism by which cholesterol affects rates and efficiency of pore formation is not fully understood, but cholesterol is known to abolish a gel phase in which SM could form in a unsaturated phospholipid bilayer (in the absence of cholesterol). In so doing, cholesterol increases diffusion in the SM-rich domain, but decreases diffusion in the unsaturated phospholipid domain $[23,24]$. Cholesterol may also affect hydrogen bonding in the membrane interface, since its polar hydroxyl can act as both hydrogen bond donor and acceptor. Palmitoyl ceramide, which interacts strongly with SM and induces gel phase formation [25,26], is known to abolish StnII-induced pore formation [21].

$\mathrm{SM}$ is a hydrogen-bonding molecule, and many of its bilayer properties (e.g., interaction with other co-lipids) are affected by hydrogen bonding $[27,28]$. Intermolecular hydrogenbonding origination from the $2 \mathrm{NH}$ function of the sphingoid base is thought to result in SM clustering in the bilayer phase [29]. Intramolecular hydrogen bonding involving the $30 \mathrm{H}$ of the sphingoid base and phosphate oxygens of the phosphocholine headgroup is very prevalent and is likely to regulate head group dynamics and orientation in SM [30,31]. The formation of StnII-pores was abolished when hydrogen bonding from both $2 \mathrm{NH}$ and $3 \mathrm{OH}$ was 
prevented by methylation [18]. This result was interpreted to be a consequence of decreased binding of toxin to methylated SM analogs. However, it is possible that intermolecular hydrogen bonding among SMs also affects the way in which pores are formed.

Most SM species have a trans $\Delta^{4}$ double bond in their long-chain base. However, dihydroSM, which lacks the unsaturation in the long-chain base, is a fairly common SM present in most cells [32,33]. Dihydro-SM is the predominant SM present in the eye-lens membrane [34]. SM and dihydro-SM are known to display very different intra- and intermolecular hydrogenbonding properties [30,31,35]. Hydrogen bonding among dihydro-SM molecules was recently shown to contribute to the lateral segregation and stabilization of a fluid dihydro-SM domain in disordered PC bilayers at high temperature [36]. A similar segregation of a fluid SM domain was not observed. The much stronger hydrogen bonding in dihydro-SM bilayers, as compared to acyl-chain matched SM bilayers, was also shown to have a fairly large effect on cholesterol and ceramide interactions with SMs [37].

Now, we have examined how pore formation by two different toxins, with highly similar structures but quite different hemolytic activities [19,38], is affected by the differing hydrogen-bonding properties of SM and dihydro-SM bilayers. We used N-palmitoyl-SM (PSM) or dihydro-PSM in mixed bilayers with 1-palmitoyl-2-oleoyl-sn-glycero-3-phosphocholine (POPC) (1:4 molar ratio) and $N$-oleoyl-SM (OSM) or dihydro-OSM in pure SM bilayers (the latter was possible because of the low gel-liquid crystalline-phase transition temperature of OSM/dihydro-OSM [39]). Our results show that StnII binding to bilayers and the subsequent pore formation were dramatically attenuated in bilayers containing dihydro-SM bilayers as compared to SM-containing bilayers. The addition of benzyl alcohol rescued the pore formation process in dihydro-SM bilayers, possibly by rearranging interfacial hydrogen bonding in a way that favored pore formation also in dihydro-OSM bilayers. We speculate that also other protein-driven processes in membranes may be affected by strong interfacial hydrogen bonding among dihydro-SM molecules.

\section{Materials and methods}

\subsection{Materials}

POPC, egg SM, sphingosyl-phosphorylcholine (lyso SM), sphinganin-phosphorylcholine (lyso dihydro-SM), and OSM were obtained from Avanti Polar Lipids (Alabaster, AL, USA). PSM 
was purified from egg SM as described previously [40]. Dihydro-PSM was prepared from PSM by hydrogenation using $\mathrm{H}_{2}$-gas and palladium (10\%) on charcoal (Sigma-Aldrich, St. Louis, MO, USA) as a catalyst, as described previously [39]. Dihydro-OSM was prepared from oleic acid anhydride (Sigma-Aldrich) and sphinganin-phosphorylcholine, as described previously [41]. Benzyl alcohol and calcein were obtained from Sigma-Aldrich. DPH was obtained from Molecular Probes (Eugene OR, USA). StnI and StnII were produced in an E.coli expression system and purified, again, as previously [38].

\subsection{Calcein release from $L U V S$}

Calcein-entrapped large unilamellar vesicles (LUVs) were prepared from POPC/PSM and POPC/dihydro-PSM (4:1 molar ratio) and from pure OSM and dihydro-OSM by extrusion through 200-nm filters at $60^{\circ} \mathrm{C}$ [18]. Briefly, the desired lipids were mixed and dried under a stream of nitrogen. Prior to extrusion, the dry lipid films were hydrated for $30 \mathrm{~min}$ at $60{ }^{\circ} \mathrm{C}$ in Tris buffer (10 mM Tris, $140 \mathrm{mM} \mathrm{NaCl}, \mathrm{pH}$ 7.4) containing calcein. The calcein concentration was $100 \mathrm{mM}$, and the final lipid concentration was $1.25 \mathrm{mM}$. LUVs were separated from nonentrapped calcein by gel filtration on Sephacryl S200HR. The LUVs were used for permeabilization studies within $12 \mathrm{~h}$. The concentrations of LUV and StnII during calcein leakage experiments were $2.5 \mu \mathrm{M}$ and $20 \mathrm{nM}$, respectively. Emission at $550 \mathrm{~nm}$ was followed at $23^{\circ} \mathrm{C}$ as a function of time (Ex $\left.480 \mathrm{~nm}\right)$. Fluorescence emission was measured in a PTI Quanta-Master spectrofluorimeter (Photon Technology International, Inc. NJ, USA). The released fraction of calcein was determined based on the maximum calcein release induced by Triton X-100. To ensure that no spontaneous leakage occurred, the emission was measured for each sample for $5 \mathrm{~min}$ before addition of toxin. A steady signal level, indicating intact vesicles, was observed for all samples. When benzoyl alcohol was used, it was added to preformed LUVs 30 min prior to initiation of experiments.

\subsection{Surface plasmon resonance measurements}

The association of StnII with lipid bilayers on coated gold chips was performed as follows. LUVs were prepared from OSM and dihydro-OSM in Tris buffer by extrusion through $100 \mathrm{~nm}$ polycarbonate filters at $60^{\circ} \mathrm{C}$. StnII binding to the coated bilayers was studied at $23^{\circ} \mathrm{C}$ with a BioNavis SPR Navi 200 instrument (BioNavis Ltd, Tampere, Finland). The sensor gold chip 
was coated with a carboxymethylated dextran layer treated with $\mathrm{N}$-hydroxysuccinimide and $\mathrm{N}$-ethyl-N'(dimethylaminopropyl) carbodiimide to activate the surface for capture of phospholipid membranes [18]. All solutions used for SPR were filtered through $0.2 \mu \mathrm{m}$ membrane filters and degassed by bath sonication before use. The running buffer was $10 \mathrm{mM}$ Tris, $140 \mathrm{mM} \mathrm{NaCl}, \mathrm{pH} 7.4$, and the flow rate was $5 \mu \mathrm{l} / \mathrm{min}$.

First, the chip surface was cleaned with two injections of 10 mM CHAPS. Then, extruded LUVs ( $0.5 \mathrm{mM}$ lipid concentration) were applied on the surface (10 min injection) and unbound vesicles were removed by one ( $2 \mathrm{~min}$ ) injection of $50 \mathrm{mM} \mathrm{NaOH}$. Bovine serum albumin (0.1 mg/ml, $2 \mathrm{~min}$ injection) was used to verify that the chip did not have uncovered areas. Finally StnII $(1.0 \mu \mathrm{M})$ was applied for $10 \mathrm{~min}$, after which buffer alone was injected for 2 min to study toxin dissociation. The chip was regenerated with CHAPS, as in the beginning of the experiment.

\section{4. $\zeta$-potential measurements}

To measure toxin/LUV bilayer interactions, vesicles were prepared from OSM and dihydroOSM in Tris buffer ( $\mathrm{pH} 7.4,140 \mathrm{mM} \mathrm{NaCl}$ ). They were exposed to increasing concentrations of StnII for $20 \mathrm{~min}$ prior to measuring the $\zeta$-potential of the aggregates. A Malvern Zetasizer ZS (Worcestershire, UK) was used for the measurements. Values given are averages \pm SD of $n=3$.

\subsection{Interaction of StnII with OSM or dihydro-OSM monolayers}

To measure the penetration of StnII into OSM and dihydro-OSM monolayers, these were spread to an initial surface pressure of $9 \mathrm{mN} / \mathrm{m}$. Following injection of StnII into the buffer subphase of the monolayers ( $0.8 \mu \mathrm{M}$ final concentration), which were kept at constant surface area, the penetration of toxin into the monolayer led to a time-dependent increase in lateral surface pressure [42]. The experiments were repeated three times, and a set of representative penetration curves are shown.

\subsection{Effects of benzyl alcohol on bilayer fluidity}


To determine the bilayer acyl chain order in the presence of increasing amounts of benzyl alcohol (BA), steady state fluorescence anisotropy of DPH was measured on a PTI QuantaMaster spectrofluorimeter operating in the T-format, essentially following the procedure described in [43]. Briefly, OSM and dihydro-OSM LUVs were prepared and exposed to increasing amounts of BA for $30 \mathrm{~min}$. After this period, DPH anisotropy was measured at the indicated temperature as a function of BA concentration. DPH was used at $1 \mathrm{~mol} \%$ of the total lipid. The wave lengths of excitation and emission of DPH were $360 \mathrm{~nm}$ and $430 \mathrm{~nm}$, respectively. The steady state anisotropy was calculated as described previously [44].

\section{Results}

\subsection{Bilayer permeabilization by sticholysins}

We have examined pore formation by StnI and StnII in bilayers containing SM and dihydroSM. It has previously been shown that interfacial hydrogen bonding is very different in SM and dihydro-SM containing bilayers [30,31,35,36]. This difference results mainly from the increased flexibility of the $30 \mathrm{H}$ in the long-chain base of dihydro-SM $[37,45]$. Our main aim was to study how such strong intermolecular hydrogen bonding (in dihydro-SM) may affect sticholysin-induced pore formation and membrane permeabilization. Pore formation was assessed by measuring calcein release from large unilamellar vesicles (200 nm diameter). The binding of both StnI and StnII to bilayers is mediated by SM, which acts as a recognition target for the pore-forming toxins. After monomer binding to the bilayer, an oligomerization process takes place in the membrane, followed eventually by the formation of a pore that allows calcein to be released from the entrapped volume within the LUVs. Pore formation by sticholysins is concentration-dependent. We used a concentration of $20 \mathrm{nM}$, which corresponds to a lipid:StnII (or StnI) ratio of about 125:1. As shown in figure 1A, the addition of StnI or StnII to LUVs prepared from POPC and PSM (4:1 molar ratio) led to a timedependent release of calcein from the vesicles. The rate and extent of pore formation was more efficient with StnI than with StnII. However, when the LUVs contained dihydro-PSM instead of PSM, the pore formation by both StnI and StnII was very low and hardly above background leakage (Fig. 1A) under the conditions used. The temperature for the experiment shown in Figure $1 \mathrm{~A}$ was $23^{\circ} \mathrm{C}$. At this temperature the SM is more ordered than the POPC, but a gel phase is not present [46]. When LUVs were made of pure OSM or pure dihydro-OSM (Fig. 
1B), a similar calcein release pattern, induced by StnII, was observed as for the saturated SMs. StnII efficiently formed pores in OSM bilayers, which led to calcein release, but the pore formation and subsequent calcein release was much less efficient in dihydro-OSM bilayers, under the conditions used. The calcein release from OSM and dihydro-OSM LUVs was performed at $24^{\circ} \mathrm{C}$ and $28{ }^{\circ} \mathrm{C}$, respectively. At these temperatures, the bilayers are fluid and the acyl chain order in the two populations of LUVs is the same ([37]]; see also Fig. 6).

\subsection{Analysis of bilayer association of StnII, using SPR, $\zeta$-potential measurements, or a} competition assay

To further assess StnII binding to and pore formation in bilayers formed from OSM and dihydro-OSM, we next performed surface plasmon resonance measurements. As shown in figure 2, the SPR sensogram indicated efficient binding of StnII to and accumulation in bilayers formed from OSM. However, StnII binding to bilayers made from dihydro-OSM was markedly attenuated (Fig. 2). The SPR data are consistent with the very low rate of calceinrelease from dihydro-OSM vesicles after exposure to StnII.

Since StnII is a positively charged toxin at pH 7 (the isoelectric point of StnII is above 9.5 [19]), we measured StnII/LUV interaction based on $\zeta$-potential measurements. SM is a zwitter-ionic phospholipid, and LUVs prepared from SM should have a net charge at $\mathrm{pH} 7$, which is close to zero. In fact, the LUVs had a small negative overall charge $(-1.4 \mathrm{mV}$ and -2.1 $\mathrm{mV}$ for OSM and dihydro-OSM LUVs at pH 7, respectively Fig. 3). The addition of StnII to LUVs prepared from OSM led to a concentration-dependent increase in the net charge (positive). However, with LUVs containing dihydro-OSM, StnII addition did not result in the accumulation of the positively charged StnII in the LUVs. Instead a small decrease in the overall dihydro-OSM LUV charge was observed (Fig. 3).

Finally, we measured calcein-release from OSM vesicles (with constant ratio of calcein vesicles and StnII), in the presence of increasing amounts of unlabelled LUVs prepared from either OSM or dihydro-OSM. Since StnII also should bind to unlabelled OSM LUVs, the effective ratio of calcein-entrapped LUVs and StnII should decrease with increasing presence of unlabelled OSM vesicles, and the amount of calcein-release should decrease. The same should happen in the presence of dihydro-OSM LUVs if StnII bound to these bilayers. The results in figure 4 show a concentration-dependent reduction in maximum calcein release when OSM 
vesicles were added. However, StnII failed to bind similarly to dihydro-OSM vesicles, and hence the effect of unlabelled dihydro-OSM vesicles on calcein release from OSM LUVs was small. Clearly, the binding of StnII to LUVs was very different according to whether they were prepared from OSM or dihydro-OSM. Altogether, the results obtained with StnII (Figs. 1-4) suggest that binding to dihydro-SM, and/or oligomerization to functional pores is substantially compromised when compared to binding and pore-formation in SM-containing bilayers.

\subsection{Monolayer penetration of StnII}

Stn is known to bind to monolayers containing SM [42], and if the monolayer is kept at constant area, its lateral surface pressure will increase as more toxin accumulates in the monolayer. We examined StnII interaction with OSM and dihydro-OSM monolayers at the air/buffer interface. The monolayers were spread to a lateral surface pressure of $9 \mathrm{mN} / \mathrm{m}$, and after 5 min equilibration time, StnII (final subphase concentration $0.8 \mu \mathrm{M}$ ) was injected into rthe stirred subphase. The initial kinetics of toxin penetration into both OSM and dihydro-OSm bilayers was similar (Fig.5). However, after reaching a surface pressure or about $28 \mathrm{mN} / \mathrm{m}$, the toxin penetration into the dihydro-OSM monolayer ceased and the monolayer surface pressure stabilized at $30 \mathrm{mN} / \mathrm{m}$ after $10 \mathrm{~min}$ of toxin exposure. With OSM monolayers, toxin penetration continued to higher surface pressures when compared to dihydro-OSM monolayers, and after $10 \mathrm{~min}$ a surface pressure of about $34 \mathrm{mN} / \mathrm{m}$ was reached. These results suggest that as packing became tighter (at increased surface pressure), intermolecular hydrogen bonding among dihydro-OSM molecules became stronger (compared to the situation in OSM monolayers) and toxin penetration ceased at a lower effective surface pressure.

\subsection{Does interference with interfacial hydrogen bonding affect StnII-induced pore formation?}

Finally, to further examine how hydrogen bonding in the SM domains affected StnII membrane binding and subsequent pore formation, we used increasing concentrations of BA to interfere with interfacial hydrogen bonding in OSM and dihydro-OSM LUV bilayers. We observed that whereas BA enhanced StnII-induced pore formation in dihydro-OSM bilayers (Fig. 6), it did not show a similar stimulatory effect on StnII-induced pore formation in OSM 
LUV bilayers, under the conditions used (suggesting that pore formation with OSM at the toxin concentration used was not hydrogen-bonding limited). In addition to affecting hydrogen bonding at the bilayer interface, BA also affected acyl chain order, as determined from DPH anisotropy measurements (Fig. 7). However, the concentration-dependent effect of BA on acyl chain order was equal in both (OSM and dihydro-OSM) bilayers.

\section{Discussion}

In this study we present evidence that Stn interaction with SM membranes is very different from Stn interaction with dihydro-SM membranes. The differences were clearly revealed using different experimental approaches: (i) we observed much slower Stn-induced calcein release from dihydro-SM bilayers than from SM bilayers (Fig. 1); (ii) we measured much less total binding of StnII to supported bilayers (SPR, Fig. 2) and LUVs ( $\zeta$-potential, Fig. 3) prepared from dihydro-OSM as compared to OSM bilayers; (iii) dihydro-OSM vesicles failed to efficiently compete with OSM vesicles for StnII binding, as determined from a competition assay (Fig.4); (iv) StnII penetration into dihydro-OSM monolayers was less efficient at higher surface pressures as compared to OSM monolayers (Fig.5). Our working hypothesis is that the differing intermolecular hydrogen bonding properties of SM and dihydro-SM may explain all our results.

Let us first review the literature evidence that hydrogen bonding among dihydro-SMs is stronger than (or at least different from) that between SMs. The first studies on the properties of SM and dihydro-SM by FTIR and NMR suggested that both intra- and intermolecular hydrogen bonding was different in bilayers prepared from saturated SM and from dihydro-SM [30,31]. Further, although PSM and dihydro-PSM occupy the same mean molecular area in monolayer membranes, their gel-liquid crystalline-phase transition temperature is different by about $6.5^{\circ} \mathrm{C}$ (higher for dihydro-PSM [47]), suggesting a hydrogen-bonding stabilization of the gel phase of dihydro-SM. It was also noted that the rotational correlation time of $N$-DPHSM was different for PSM and dihydro-PSM bilayers in their fluid phase, and that Prodan partitioning into dihydro-PSM bilayers, as compared to PSM bilayers, was much reduced [47]. Such observations are most likely explained by differing hydrogen-bonding properties of SM and dihydro-SM bilayers.

Furthermore, Kinoshita and coworkers have recently demonstrated that dihydro-18:0-SM shows liquid/liquid phase immiscibility in di-oleoyl-PC bilayers above $60{ }^{\circ} \mathrm{C}$, which was not 
seen in 18:0-SM/di-oleoyl-PC bilayers [36]. Again, stronger hydrogen bonding in dihydro-SM bilayers was cited as the most likely reason for such a thermostable liquid phase. Finally, we showed that the lateral segregation of dipalmitoyl glycerol is much more enhanced in dihydro-OSM bilayers than in OSM bilayers at equal acyl chain order [37]. It appeared that strong intermolecular hydrogen bonding among dihydro-OSM bilayers caused the segregation of dipalmitoyl glycerol into its own phase, whereas the monomer solubility of dipalmitoyl glycerol in OSM bilayers was much less affected (at comparable bilayer compositions and temperature). All these previous observations are compatible with the present findings, that pore formation by StnI and StnII was markedly different in SM and dihydro-SM bilayers.

Stn-induced calcein release was markedly attenuated in dihydro-SM containing bilayers. This finding could results from weaker Stn monomer binding to bilayers, or from hindered lateral diffusion, oligomerization, and pore formation in dihydro-SM containing bilayers. Both processes are likely to be affected by strong intermolecular hydrogen bonding among dihydro-SM molecules. However, we cannot exclude the possibility that the $30 \mathrm{H}$ in SM would be closer to the interface, as compared to the $30 \mathrm{H}$ in dihydro-SM, since the trans double bond has a partly polar nature when compared to a single $\mathrm{C}-\mathrm{C}$ bond. A deeper localisation of the $30 \mathrm{H}$ in dihydro-SM could possiby hinder Stn association with the bilayer interface in a way observed here.

Our SPR and $\zeta$-potential measurements suggest that total StnII binding to bilayers was reduced in dihydro-SM bilayers. It is therefore possible that strong intermolecular hydrogen bonding between dihydro-SM molecules can increase the energy barrier for toxin penetration into the interfacial region of the bilayer. Support for this interpretation was given by our monolayer experiments, which showed that StnII penetration into dihydro-OSM monolayers was less efficient at high lateral surface pressures, when compared to OSM monolayers.

Additional support for the involvement of intermolecular hydrogen bonding in regulating membrane association of StnII is given by our BA data. The hydroxyl group of BA allows it to participate in hydrogen bonding similarly as cholesterol. Cholesterol is known to facilitate pore formation by different actinoporins [8,21,48,49]. BA showed a strong activation of StnIIdependent calcein release from dihydro-OSM bilayers. This effect was not dependent on altered packing properties in the bilayer, as evidenced by the similar effects of BA on acyl chain order in both OSM and dihydro-OSM bilayers. OSM bilayers were not affected by BA under the conditions used, probably because pore formation was already maximal and could not further be enhanced. We have observed that BA is capable of enhancing StnII-induced 
calcein release in any SM-containing bilayer, provided that sub-saturation levels of toxins are used (data not shown). It has previously been shown that EqtII-induced calcein release from LUVs is facilitated by different alkanols [50]. It is likely that the effect of alkanols, BA, and cholesterol on pore formation by actinoporins could be due to effects of the compounds on interfacial hydrogen bonding in SM-containing bilayers. However, we cannot rule out that the free hydroxyl on these compounds could interact with Stn and somehow stabilize the membrane association of different actinoporins.

To conclude, we have shown that pore formation by sticholysins are markedly restricted in bilayers containing dihydro-SM when compared to acyl-chain-matched normal SM. We have preliminary evidence that similar restrictions apply to EqtII-induced pore formation in dihydro-SM-containing bilayers (data not shown). We would not be surprised if other diffusion related or bilayer penetration processes by other proteins are similarly affected by strong intermolecular hydrogen bonding in dihydro-SM-containing bilayers. In fact, it has been observed that enrichment of dihydro SM in cells reduced infectivity of the HIV-1 virus, a process which likely was sensitive to interactions among the dihydro-SMs [51].

\section{Author contribution}

All authors contributed in the execution and completion of the study and in the writing of the manuscript. All authors have seen and accepted the final submitted version of the manuscript.

\section{Acknowledgments}

The work was funded by generous grants: BFU2012-32404 from the Spanish Ministerio de Ciencia e Innovación (JGG and AMP), a FPU fellowship grant (S.G.-L); the Academy of Finland, the Sigrid Juselius Foundation, and the Åbo Akademi Foundation (JPS). TY was supported in part by the International Collaboration Promotion Program from Osaka University.

\section{References}

[1] J. Alegre-Cebollada, M. Oñaderra, J.G. Gavilanes, A. Martínez-del-Pozo, Sea anemone actinoporins: the transition from a folded soluble state to a functionally active membrane-bound oligomeric pore, Curr Protein Pept Sci 8 (2007) 558-572. 
[2] G. Anderluh and P. Maček, Cytolytic peptide and protein toxins from sea anemones (Anthozoa: Actiniaria), Toxicon 40 (2002) 111-124.

[3] B. Bakrač and G. Anderluh, Molecular mechanism of sphingomyelin-specific membrane binding and pore formation by actinoporins, Adv Exp Med Biol 677 (2010) 106115.

[4] P. Maček, Polypeptide cytolytic toxins from sea anemones (Actiniaria), FEMS Microbiol Immunol 5 (1992) 121-129.

[5] N. Rojko, S.M. Dalla, P. Maček, G. Anderluh, Pore formation by actinoporins, cytolysins from sea anemones, Biochim Biophys Acta (2015). doi: 10.1016/j.bbamem.2015.09.007.

[6] G. Anderluh and P. Maček, Dissecting the actinoporin pore-forming mechanism, Structure. 11 (2003) 1312-1313.

[7] L. Garcia-Ortega, J. Alegre-Cebollada, S. García-Linares, M. Bruix, A. Martínez-del-Pozo, J.G. Gavilanes, The behavior of sea anemone actinoporins at the water-membrane interface, Biochim Biophys Acta 1808 (2011) 2275-2288.

[8] V. De Los Ríos, J.M. Mancheño, M.E. Lanio, M. Oñaderra, J.G. Gavilanes, Mechanism of the leakage induced on lipid model membranes by the hemolytic protein sticholysin II from the sea anemone Stichodactyla helianthus, Eur J Biochem 252 (1998) 284289.

[9] M. Tejuca, M.D. Serra, M. Ferreras, M.E. Lanio, G. Menestrina, Mechanism of membrane permeabilization by sticholysin I, a cytolysin isolated from the venom of the sea anemone Stichodactyla helianthus, Biochemistry 35 (1996) 14947-14957.

[10] W.R. Kem and B.M. Dunn, Separation and characterization of four different amino acid sequence variants of a sea anemone (Stichodactyla helianthus) protein cytolysin, Toxicon 26 (1988) 997-1008.

[11] I. Ferlan and D. Lebez, Equinatoxin, a lethan protein from Actinia equina - purification and characterization, Toxicon 12 (1974) 57-61. 
[12] A.E. Mechaly, A. Bellomio, K. Morante, J.M. González-Mañas, D.M. Guérin, Crystallization and preliminary crystallographic analysis of fragaceatoxin $\mathrm{C}$, a pore-forming toxin from sea anemone Actinia fragacea, Acta Crystallogr Sect F 65 (2009) 357360 .

[13] A. Athanasiadis, G. Anderluh, P. Maček, D. Turk, Crystal structure of the soluble form of equinatoxin II, a pore-forming toxin from the sea anemone Actinia equina, Structure. 9 (2001) 341-346.

[14] S. García-Linares, I. Castrillo, M. Bruix, M. Menendez, J. Alegre-Cebollada, A. Martínez-delPozo, J.G. Gavilanes, Three-dimensional structure of the actinoporin sticholysin I. Influence of long-distance effects on protein function, Arch Biochem Biophys 532 (2013) 39-45.

[15] J.M. Mancheño, J. Martin-Benito, M. Martinez-Ripoll, J.G. Gavilanes, J.A. Hermoso, Crystal and electron microscopy structures of sticholysin II actinoporin reveal insights into the mechanism of membrane pore formation, Structure. 11 (2003) 13191328.

[16] K. Tanaka, J.M. Caaveiro, K. Morante, J.M. Gonzalez-Manas, K. Tsumoto, Structural basis for self-assembly of a cytolytic pore lined by protein and lipid, Nat Commun 6 (2015) p.6337.

[17] U. Ros and A.J. Garcia-Saez, More Than a Pore: The Interplay of Pore-Forming Proteins and Lipid Membranes, J Membr Biol 248 (2015) 545-561.

[18] T. Maula, Y.J.E. Isaksson, S. Garcia-Linares, S. Niinivehmas, O.T. Pentikainen, V. Kurze, S. Yamaguchi, T. Yamamoto, S. Katsumura, J.G. Gavilanes, A. Martínez-del-Pozo, J.P. Slotte, $2 \mathrm{NH}$ and $30 \mathrm{H}$ are crucial strctural requirements in sphingomyelin for sticholysin II binding and pore formation in bilayer membranes, Biochim Biophys Acta 1828 (2013) 1390-1395.

[19] C. Alvarez, J.M. Mancheno, D. Martinez, M. Tejuca, F. Pazos, M.E. Lanio, Sticholysins, two pore-forming toxins produced by the Caribbean Sea anemone Stichodactyla helianthus: their interaction with membranes, Toxicon 54 (2009) 1135-1147. 
[20] B. Bakrač, I. Gutierrez-Aguirre, Z. Podlesek, A.F. Sonnen, R.J. Gilbert, P. Maček, J.H. Lakey, G. Anderluh, Molecular determinants of sphingomyelin specificity of a eukaryotic pore-forming toxin, J Biol Chem 283 (2008) 18665-18677.

[21] I. Alm, S. García-Linares, J.G. Gavilanes, A. Martínez-del-Pozo, J.P. Slotte, Cholesterol stimulates and ceramide inhibits Sticholysin II-induced pore formation in complex bilayer membranes, Biochim Biophys Acta 1848 (2015) 925-931.

[22] M.Z. Islam, J.M. Alam, Y. Tamba, M.A. Karal, M. Yamazaki, The single GUV method for revealing the function of antimicrobial, pore-forming toxin, and cell-penetrating peptides or proteins, Phys Chem Chem Phys 14 (2014) 15752-15767.

[23] A. Filippov, G. Oradd, G. Lindblom, Lipid lateral diffusion in ordered and disordered phases in raft mixtures, Biophys J 86 (2004) 891-896.

[24] A. Filippov, G. Oradd, G. Lindblom, Sphingomyelin structure influences the lateral diffusion and raft formation in lipid bilayers, Biophys J 90 (2005) 2086-92

[25] L. Silva, R.F. de Almeida, A. Fedorov, A.P. Matos, M. Prieto, Ceramide-platform formation and -induced biophysical changes in a fluid phospholipid membrane, Mol Membr Biol. 23 (2006) 137-148.

[26] I. Artetxe, C. Sergelius, M. Kurita, S. Yamaguchi, S. Katsumura, J.P. Slotte, T. Maula, Effects of sphingomyelin headgroup size on interactions with ceramide, Biophys J 104 (2013) 604-612.

[27] Y. Barenholz and T.E. Thompson, Sphingomyelins in bilayers and biological membranes, Biochimica Biophysica Acta 604 (1980) 129-158.

[28] M. Lonnfors, J.P. Doux, J.A. Killian, T.K. Nyholm, J.P. Slotte, Sterols Have Higher Affinity for Sphingomyelin than for Phosphatidylcholine Bilayers even at Equal AcylChain Order, Biophys J 100 (2011) 2633-2641.

[29] E. Mombelli, R. Morris, W. Taylor, F. Fraternali, Hydrogen-bonding propensities of sphingomyelin in solution and in a bilayer assembly: a molecular dynamics study, Biophys J 84 (2003) 1507-1517. 
[30] S.R. Ferguson-Yankey, D. Borchman, K.G. Taylor, D.B. DuPre, M.C. Yappert, Conformational studies of sphingolipids by NMR spectroscopy. I. Dihydrosphingomyelin, Biochim Biophys Acta 1467 (2000) 307-25.

[31] C.M. Talbott, I. Vorobyov, D. Borchman, K.G. Taylor, D.B. DuPre, M.C. Yappert, Conformational studies of sphingolipids by NMR spectroscopy. II. Sphingomyelin, Biochim Biophys Acta 1467 (2000) 326-37.

[32] J.W. Kok, M. Nikolova-Karakashian, K. Klappe, C. Alexander, A.H. Merrill, Jr., Dihydroceramide biology. Structure-specific metabolism and intracellular localization, J Biol Chem 272 (1997) 21128-36.

[33] P.B. Schneider and E.P. Kennedy, Metabolism of labeled dihydrosphingomyelin in vivo, J Lipid Res 9 (1968) 58-64.

[34] D. Borchman and M.C. Yappert, Lipids and the ocular lens, J Lipid Res 51 (2010) 24732488.

[35] R.M. Epand, Cholesterol in bilayers of sphingomyelin or dihydrosphingomyelin at concentrations found in ocular lens membranes, Biophys J 84 (2003) 31023110.

[36] M. Kinoshita, N. Matsumori, M. Murata, Coexistence of two liquid crystalline phases in dihydrosphingomyelin and dioleoylphosphatidylcholine binary mixtures, Biochim Biophys Acta 1838 (2014) 1372-1381.

[37] T. Yasuda, Md.A. Al Sazzad, N.Z. Jäntti, O.T. Pentikäinen, J.P. Slotte, The influence of hydrogen bonding on sphingomyelin/colipid interactions in bilayer membranes., Biophys J (in press) (2016).

[38] J. Alegre-Cebollada, G. Clementi, M. Cunietti, C. Porres, M. Oñaderra, J.G. Gavilanes, A.M. Pozo, Silent mutations at the 5 -end of the cDNA of actinoporins from the sea anemone Stichodactyla helianthus allow their heterologous overproduction in Escherichia coli, J Biotechnol 127 (2007) 211-221. 
[39] M. Kuikka, B. Ramstedt, H. Ohvo-Rekilä, J. Tuuf, J.P. Slotte, Membrane properties of Derythro-N-acyl sphingomyelins and their corresponding dihydro species, Biophys J 80 (2001) 2327-2337.

[40] B. Terova, J.P. Slotte, T.K. Nyholm, Miscibility of acyl-chain defined phosphatidylcholines with N-palmitoyl sphingomyelin in bilayer membranes, Biochim Biophys Acta 1667 (2004) 182-189.

[41] R. Cohen, Y. Barenholz, S. Gatt, A. Dagan, Preparation and characterization of well defined D-erythro sphingomyelins, Chem Phys Lipids 35 (1984) 371-384.

[42] L. Pedrera, M.L. Fanani, U. Ros, M.E. Lanio, B. Maggio, C. Alvarez, Sticholysin I-membrane interaction: an interplay between the presence of sphingomyelin and membrane fluidity, Biochim Biophys Acta 1838 (2014) 1752-59.

[43] S. Jaikishan, A. Bjorkbom, J.P. Slotte, Sphingomyelin analogs with branched N-acyl chains: The position of branching dramatically affects acyl chain order and sterol interactions in bilayer membranes, Biochim Biophys Acta 1798 (2010) 19871994.

[44] J.R. Lakowicz, Principles of Fluorescence Spectroscopy, Kluwer Academic / Plenum Publishers, New York, 1999.

[45] J.P. Slotte, The importance of hydrogen bonding in sphingomyelin's membrane interactions with co-lipids, Biochim Biophys Acta 1858 (2015) 304-310.

[46] V. Lukacova, M. Peng, R. Tandlich, A. Hinderliter, S. Balaz, Partitioning of organic compounds in phases imitating the headgroup and core regions of phospholipid bilayers, Langmuir 22 (2006) 1869-1874.

[47] T. Nyholm, M. Nylund, A. Soderholm, J.P. Slotte, Properties of palmitoyl phosphatidylcholine, sphingomyelin, and dihydrosphingomyelin bilayer membranes as reported by different fluorescent reporter molecules, Biophys J 84 (2003) 987-997. 
[48] J. Alegre-Cebollada, A. Martínez-del-Pozo, J.G. Gavilanes, E. Goormaghtigh, Infrared spectroscopy study on the conformational changes leading to pore formation of the toxin sticholysin II, Biophys J 93 (2007) 3191-3201.

[49] D. Martinez, A. Otero, C. Alvarez, F. Pazos, M. Tejuca, L.M. Eliana, I. Gutierrez-Aguirre, A. Barlic, I. Iloro, A.J. Luis, J.M. Gonzalez-Manas, E. Lissi, Effect of sphingomyelin and cholesterol on the interaction of St II with lipidic interfaces, Toxicon 49 (2007) 68-81.

[50] P. Maček, M. Zecchini, K. Stanek, G. Menestrina, Effect of membrane-partitioned nalcohols and fatty acids on pore-forming activity of a sea anemone toxin, Eur Biophys J 25 (1997) 155-162.

[51] C.R. Vieira, J.M. Munoz-Olaya, J. Sot, S. Jimenez-Baranda, N. Izquierdo-Useros, J.L. Abad, B. Apellaniz, R. Delgado, J. Martinez-Picardo, A. Alonso, J. Casas, J.L. Nieva, G. Fabrias, S. Manes, F.M. Goni. Dihydrosphingomyelin impairs HIV-1 infection by rigidifying liquid-ordred membrane domains. Chem Biol 17 (2010) 766-775. 
Figures 1-7

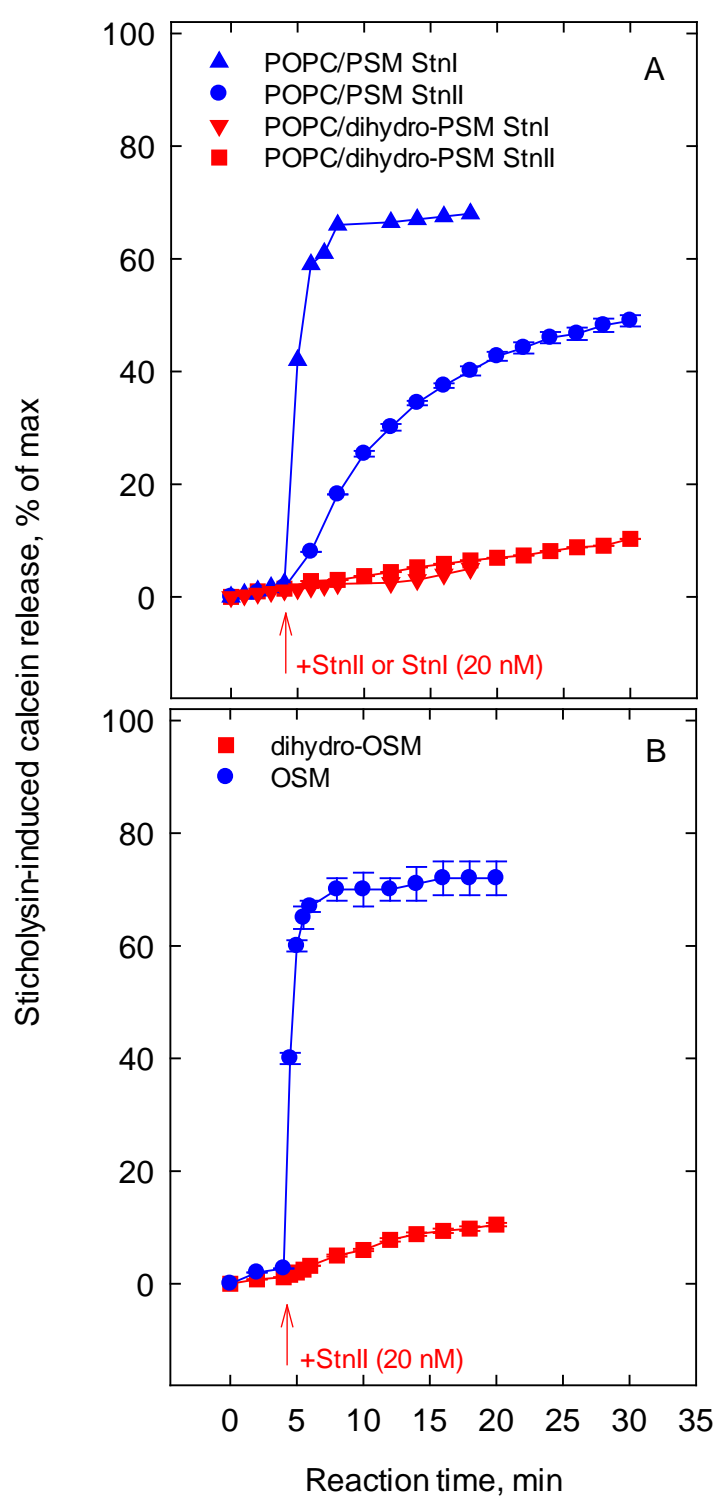

Figure 1. Calcein release by Stnl or Stnll from SM or dihydro-SM LUVs. $200 \mathrm{~nm}$ LUVs were prepared either from POPC/PSM or POPC/dihydro-PSM (4:1 molar ratio; panel A, $\left.37^{\circ} \mathrm{C}\right)$, or pure OSM $\left(24^{\circ} \mathrm{C}\right)$ or dihydro-OSM $\left(28^{\circ} \mathrm{C}\right)$ (panel B). At the indicated time (red arrow $1 \mathrm{n}$ figure), Stnl (Panel A) or Stnll was added to give final concentration of $20 \mathrm{nM}$. The total lipid:Stnll molar ratio was about 125:1. Each value is the average \pm SEM from $n=2$. 




Figure 2. Interaction of Stnll with supported bilayers of OSM or dihydro-OSM as analyzed by SPR. For SPR measurements, Stnll was added at time 0 , and the bilayer associatio of Stnll was determined as a function of time. The chip temperature was maintained at $23{ }^{\circ} \mathrm{C}$. Each value is average \pm SEM from $n=2$.

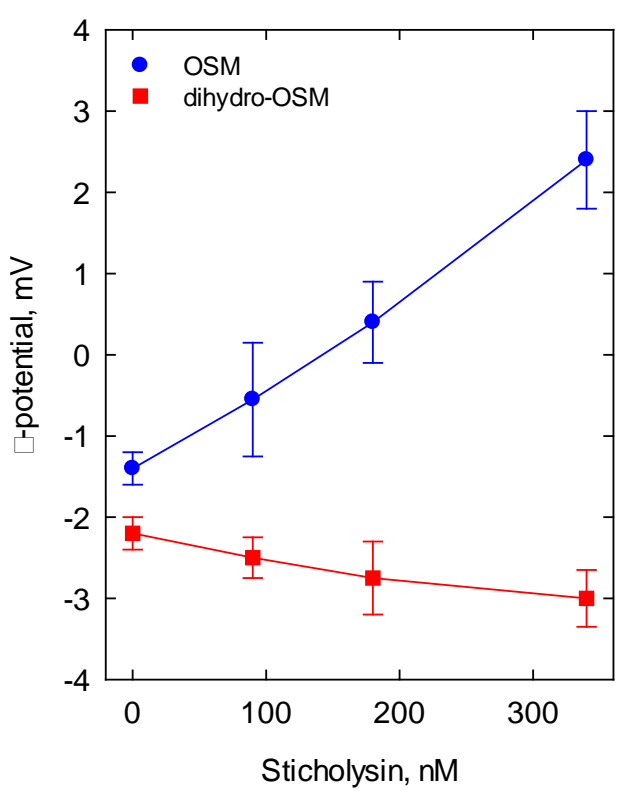

Figure 3. Interaction of StnIl with LUVs prepared from OSM and dihydro-OSM, as analyzed $\zeta$-potential. For $\zeta$-potential, $200 \mathrm{~nm}$ LUVs were exposed to indicated amounts of Stnll for $20 \mathrm{~min}$ prior to measurement of $\zeta$-potential (total lipid:StnII molar ratio 30:1 to 10:1). OSM vesicles were measured at $24^{\circ} \mathrm{C}$ and dihydro-OSM vesicles at $28^{\circ} \mathrm{C}$.Each value is average \pm SEM from $n=2$. The diameter of the LUVs were $200 \pm 20 \mathrm{~nm}$ before and after addition of Stnll. 


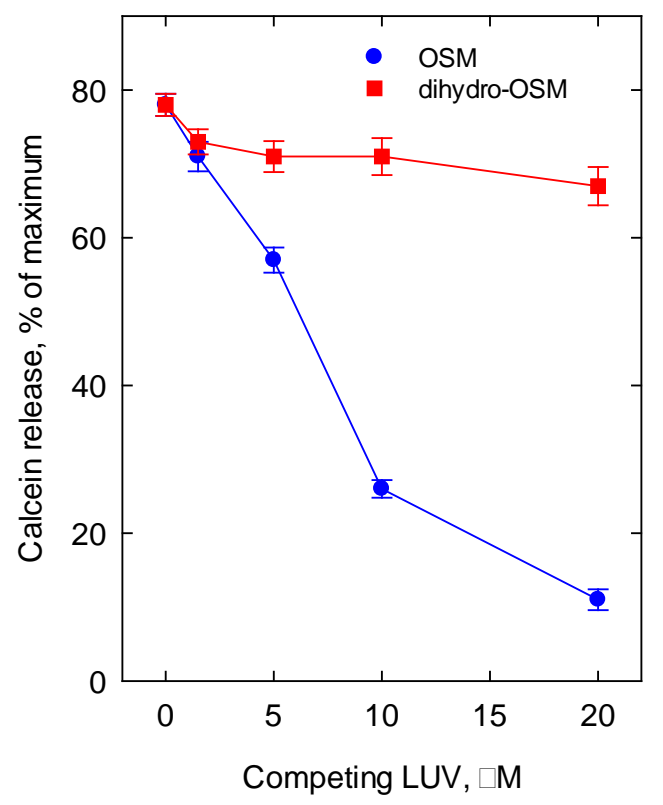

Figure 4. Competition assay for Stnll-induced calcein release from OSM LUVs. Calcein-entrapped OSM LUVs (200 nm diamater) were exposed to increasing concentrations of unlabelled OSM or dihydro-OSM LUVs ( $200 \mathrm{~nm}$ diameter) for a few minutes before Stnll was added ( $20 \mathrm{nM}$ final concentration). The maximum release of calcein after 5 min was determined. Maximum release was achieved before $5 \mathrm{~min}$ in each sample. The concentration of calcein-entrapped OSM vesicles was $3 \mu \mathrm{M}$. Each value is average \pm SEM from $n=2$. The experimental temperature was $23^{\circ} \mathrm{C}$.

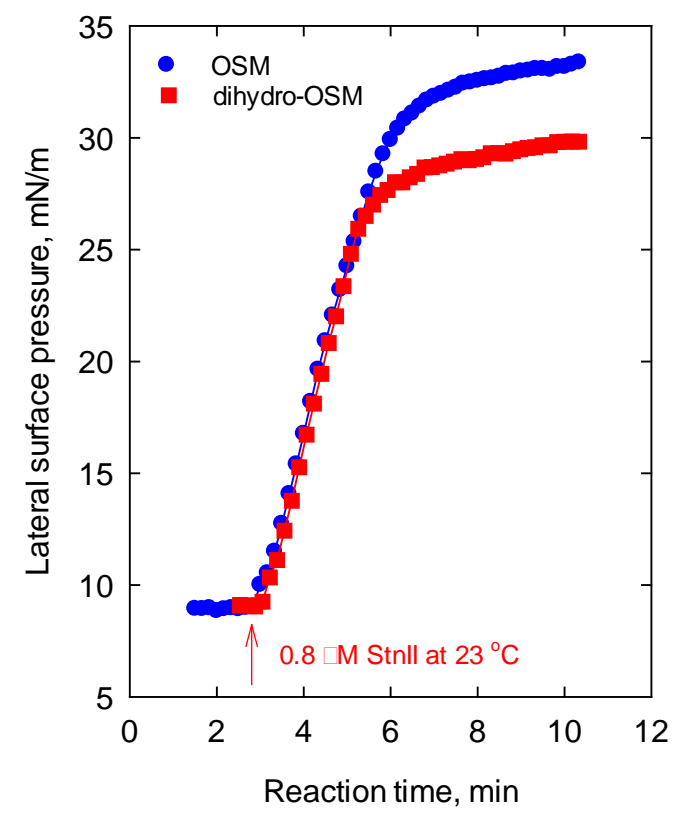

Figure 5. Penetration of Stnll into OSM or dihydro-OSM monolayers. The monolayer was spread to an initial surface pressure of about $9 \mathrm{mN} / \mathrm{m}$, and after 5 min equilibration time, Stnll was injected into the stirred buffer subphase to a 
final concentration of $0.8 \mu \mathrm{M}$. The increase in surface pressure, following toxin penetration into the monolayer, was followed as a time function at $23^{\circ} \mathrm{C}$. The curves shown are representative of three identical experiments performed with OSM or dihydro-OSM monolayers.

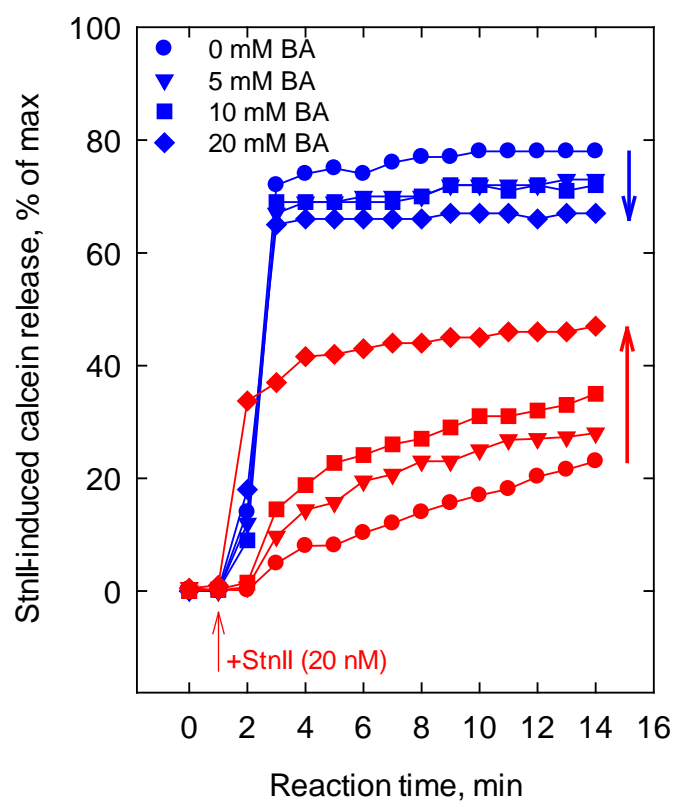

Figure 6. Effects of benzyl alcohol on Stnll-induced calcein release in LUVs prepared from OSM (blue symbols, top four lines) or dihydro-OSM (red symbols, bottom four lines). Calcein-entrapped LUVs were exposed to indicated concentrations of BA (for $30 \mathrm{~min}$ ), and then Stnll was added (red arrow). Each value is average \pm SEM from $n=2$. Identical red and blue symbols have indicated BA concentrations. 




Figure 7. Effects of benzyl alcohol on acyl chain order in LUVs prepared from OSM or dihydro-OSM. The effect of BA on acyl chain order was measured from the steady-state anisotropy of DPH in OSM and dihydro-OSM LUVs. Each value is the average \pm SEM from $n=3$. 